\title{
An Unusual Case of Extranodal Rosai-Dorfman Disease Manifesting as an Epibulbar Mass
}

\author{
Hang M. Tran ${ }^{a, b}$ Sahmon Chinichian ${ }^{a} \quad$ Kris Storkersen $^{a}$ \\ Keith Tokuhara ${ }^{a}$ \\ ${ }^{a}$ Department of Ophthalmology, Arrowhead Regional Medical Center, Colton, Calif., and \\ ${ }^{b}$ St. Mary Mercy Hospital, Livonia, Mich., USA
}

\section{Key Words}

Sinus histiocytosis with massive lymphadenopathy $\cdot$ Rosai-Dorfman disease $\cdot$ Extranodal site . Epibulbar mass

\begin{abstract}
Purpose: To report an unusual presentation of a 48-year-old man with a salmon-colored epibulbar mass, whose pathology was consistent with extranodal Rosai-Dorfman disease (RDD). Method: We conducted a retrospective and interventional case report. Results: A 48year-old white man presented with progressive left eye irritation, redness, and multiple large subcutaneous nodules on his arms and torso over the past year. He did not have any lymphadenopathy, lymphocytosis, or fever. Visual acuity and intraocular pressures were normal in both eyes. He had mild vertical diplopia on downward gaze. Slit lamp examination revealed a non-tender salmon-colored epibulbar mass of $1.0 \times 1.2 \mathrm{~cm}$. Incisional biopsy was performed. Histopathologic evaluation revealed emperipolesis, with positive CD68, positive S100, and negative CD1a staining. These findings were consistent with extranodal RDD. At the 24-month follow-up, there were no signs of recurrence, and his diplopia resolved. Conclusions: This case of RDD is rare because of the concurrent epibulbar mass with subcutaneous nodules on the torso and arms. Extranodal RDD with epibulbar mass involvement tends to be unilateral, occurring mainly in males, and evenly among people of white and black race. An epibulbar mass of any color should raise concern for systemic lymphoma and RDD. The clinical course for extranodal RDD is typically benign. Excisional biopsy is often done for diagnosis and treatment. Recurrence of the unilateral epibulbar mass after biopsy is rare, but common with bilateral epibulbar masses.


Tran et al.: An Unusual Case of Extranodal Rosai-Dorfman Disease Manifesting as an Epibulbar Mass

\section{Introduction}

Rosai-Dorfman disease (RDD) or sinus histiocytosis with massive lymphadenopathy was originally described by Destombes [1] in 1965. Four years later, Rosai and Dorfman [2] characterized it as a distinct clinicopathologic disorder based on 4 cases. Patients with this rare and nonmalignant disorder often present with painless cervical lymphadenopathy, fever, and leukocytosis. RDD often manifests in the lymph nodes of the head and neck, but it may also present in extranodal sites, such as the skin, conjunctiva, upper respiratory tract, and bone.

The pathologic findings that Rosai and Dorfman described became the basis for a new entity called 'sinus histiocytosis with massive lymphadenopathy'. These pathologic findings were [2]:

1 emperipolesis: histiocytes with engulfed lymphocytes (most common), plasma cells and erythrocytes;

2 marked fibrosis of the lymph node capsule with lymphocyte and plasma cell infiltration;

3 dilation of subcapsular and medullary septate;

4 increased intrasinusal histiocytosis with little atypia and few mitoses.

The classical findings of RDD include emperipolesis and histiocytes staining positive for S100 and CD68, and negative for CD1a.

\section{Case Report}

A 48-year-old white man presented with progressive left eye irritation, redness, and growth of multiple large subcutaneous nodules on his arms and torso over the past year. He denied any history of trauma, previous eye surgeries, intravenous drug abuse, fevers, night sweats, or unintended weight loss. His past medical history was positive for hyperlipidemia and negative for diabetes, hypertension, cancer, and lymphoproliferative diseases. His visual acuity and intraocular eye pressures were normal. Pupils were round and equally reactive, with no relative afferent papillary defect. He had full range of extraocular movement, and confrontational fields were full in both eyes. He had mild vertical diplopia on downgaze. Slit lamp examination revealed a non-tender salmon-colored epibulbar mass adherent to the inferonasal part of the left eye. Physical examination showed several 2-cm non-tender subcutaneous nodules on the torso and the left arm as well as an absence of lymphadenopathy. The basic chemistry panel, complete blood count, and erythrocyte sedimentation rate were normal. A computed tomography scan of the orbits showed a soft tissue mass measuring 1.0 $\times 1.2 \times 1.4 \mathrm{~cm}$ without intraorbital extension or bony erosion.

The epibulbar mass was biopsied to rule out malignancy (fig. 1a). The biopsy stained positive for CD68 (fig. 1b), positive for S100 (fig. 1c), and negative for CD1a. Histology showed prominent fibrosclerotic tissue with foamy vacuoles and foamy histiocytes, $25 \%$ polytypic plasma cells, multiple small benign mantle cell nodules, and emperipolesis with hematoxylin and eosin stain (fig. 1d). The pathologic features and immunostains were consistent with extranodal RDD [3] rather than with systemic lymphoma. The patient underwent surgical debulking of the epibulbar mass, with the histopathologic results matching that of the first biopsy. At the 24-month follow-up, there were no signs of recurrence, and his diplopia resolved. 
Tran et al.: An Unusual Case of Extranodal Rosai-Dorfman Disease Manifesting as an Epibulbar Mass

\section{Discussion}

The estimated incidence of RDD in the United States is 100 cases per year [4]. Ocular manifestations occur in $11.5 \%$ of RDD cases, often involving orbital soft tissues and the eyelids [5]. Infiltrations of the conjunctiva/subconjunctiva, lacrimal tract, cornea and uveal tract, as well as of the optic nerve, have been reported [5].

The epidemiology of extranodal RDD manifesting in epibulbar masses has evolved over the years. Currently, there are 20 reported cases [3, 4, 6-20]. Epibulbar mass involvement has been described as bilateral $[10,18]$. However, 14 out of 20 case reports $(70 \%)$ show unilateral involvement $[3,4,6,8,9,14,18-20]$, and 3 out of 20 case reports show the presence of lymphadenopathy $[10,14,17]$. The mean age at presentation is 30 years (standard deviation 21), which is slightly lower than the 35 years given in a previous report [3]. By sex, males are more likely affected, with 13 out of 20 case reports (65\%). There is an equal distribution between white and black race, each with 7 out of 20 case reports (35\%). Epibulbar masses are often described as pink, red, salmon-colored, and fleshy.

Our case is unique because of concurrent epibulbar mass and subcutaneous nodules on the arms and torso in the absence of lymphadenopathy, lymphocytosis, and fever. Fernandes et al. [3] examined 9 extranodal RDD cases and concluded that a lack of lymphadenopathy is characteristic for extranodal RDD. Our case report and our literature findings are consistent with the findings of Fernandes et al. [3]. Three cases in the literature report patients with epibulbar masses and subcutaneous nodules localized on the arms, legs, or anterior chest wall $[11,15,20]$. Our findings demonstrate concurrence of epibulbar mass and subcutaneous nodules that can appear anywhere on the body.

The classical findings of RDD include emperipolesis and histiocytes staining positive for S100 and CD68, and negative for CD1a. There is no standard protocol for the treatment of ocular RDD because of its rarity and the chance of spontaneous regression [2, 5]. Excisional biopsy is often done for diagnosis and treatment [3], and there is usually no recurrence after an excisional biopsy.

Consistent with the literature, our patient does not have recurrence of his unilateral epibulbar mass after excisional biopsy. Interestingly, our literature review shows that recurrence after excisional biopsies of bilateral epibulbar masses is common, appearing in 2 out of 7 cases $(29 \%)[16,17]$. This pattern has not been reported before, and recurrence may be due to coexisting uveitis in these 2 cases.

Extranodal RDD with epibulbar masses and subcutaneous nodules are exceedingly rare. Any epibulbar mass should raise concern for systemic lymphoma and RDD. The clinical course of extranodal RDD is typically benign. There is no standard protocol for treatment; however, excisional biopsy is commonly done for diagnosis and treatment. Recurrence after excisional biopsy of bilateral epibulbar masses may be due to existing uveitis, thus requiring additional surgical excision.

\section{Acknowledgements}

We would like to thank Dr. Carolyn Leach for the pathology report and the photos.

\section{Statement of Ethics}

The authors have no ethical conflicts to disclose. 
Tran et al.: An Unusual Case of Extranodal Rosai-Dorfman Disease Manifesting as an Epibulbar Mass

\section{Disclosure Statement}

The authors declare no conflicts of interest.

\section{References}

1 Destombes P: Adenitis with lipid excess, in children or young adults, seen in the Antilles and in Mali (4 cases). Bull Soc Pathol Exot Filiales 1965;58:1169-1175.

$\checkmark 2$ Rosai J, Dorfman RF: Sinus histiocytosis with massive lymphadenopathy. A newly recognized benign clinicopathological entity. Arch Pathol 1969;87:63-70.

-3 Fernandes BF, Brazuna A, Moura LR, et al: Extranodal Rosai-Dorfman disease presenting as an epibulbar tumor. Cornea 2008;27:378-381.

4 Albini TA, Evans M, See R, et al: Rosai-Dorfman disease: isolated epibulbar masses in two adult patients. Br J Ophthalmol 2005;89:241-243.

5 Foucar E, Rosai J, Dorfman R: Sinus histiocytosis with massive lymphadenopathy (Rosai-Dorfman disease): review of the entity. Semin Diagn Pathol 1990;7:19-73.

6 Al Wadani S, Robinson S, Myers R, et al: No increase in IgG4-positive plasma cells in limbal Rosai-Dorfman disease. Cornea 2014;33:844-847.

7 Cavuoto K, Galor A, Dubovy SR, et al: Subconjunctival masses associated with central nervous system RosaiDorfman disease. Cornea 2011;30:237-240.

-8 de Oliveira RC, Rigueiro M, Vieira AC, et al: Rosai-Dorfman disease manifesting as an epibulbar ocular tumour. Clin Experiment Ophthalmol 2011;39:175-177.

-9 Finger PT, Perry HD, Kempin S, et al: High-frequency ultrasound of extranodal limbal Rosai-Dorfman disease: affecting the conjunctiva, sclera, and cornea. Cornea 2007;26:888-890.

10 Ireland KC, Hutchinson AK, Grossniklaus HE: Sinus histiocytosis presenting as bilateral epibulbar masses. Am J Ophthalmol 1999;127:360-361.

11 Karcioglu ZA, Allam B, Insler MS: Ocular involvement in sinus histiocytosis with massive lymphadenopathy. Br J Ophthalmol 1988;72:793-795.

12 Maheshwari R, Shekde S: Extranodal Rosai-Dorfman disease presenting as an isolated epibulbar mass. Indian J Ophthalmol 2008;56:502-504.

13 Mellen P, Eagle R: Ocular manifestations of Rosai-Dorfman disease. Jefferson Digital Commons. 2013. http://jdc.jefferson.edu/phsrs/12/ (accessed April 30, 2015).

14 Mohadjer Y, Holds JB, Rootman J, et al: The spectrum of orbital Rosai-Dorfman disease. Ophthal Plast Reconstr Surg 2006;22:163-168.

15 Ratzinger G, Zelger B, Höbling W, et al: Sinus histiocytosis with massive lymphadenopathy Rosai-Dorfman: three unusual manifestations. Virchows Arch 2003;443:797-800.

16 Shah A, Bielory L, Mirani N, et al: Epibulbar Rosai-Dorfman disease: novel manifestation and treatment. Arch Ophthalmol 2012;130:1218-1220.

17 Tan H, Ly K: Rosai-Dorfman disease manifesting as relapsing uveitis and subconjunctival masses. Chang Gung Med J 2002;25:621-625.

18 Zimmerman LE, Hidayat AA, Grantham RL, et al: Atypical cases of sinus histiocytosis (Rosai-Dorfman disease) with ophthalmological manifestations. Trans Am Ophthalmol Soc 1988;86:113-135.

19 Stopak SS, Dreizen NG, Zimmerman LE, et al: Sinus histiocytosis presenting as an epibulbar mass. A clinicopathologic case report. Arch Ophthalmol 1988;106:1426-1428.

20 Sarwal R, Tu E, Mendelblatt FI, et al: Atypical ocular presentations of Rosai-Dorfman disease. Ocul Immunol Inflamm 2008;16:9-15. 


\section{Case Reports in \\ Ophthalmology}

\begin{tabular}{l|l}
\hline Case Rep Ophthalmol 2015;6:351-355 \\
\hline DOI: 10.1159/000440994 & $\begin{array}{l}\text { ○ 2015 The Author(s). Published by S. Karger AG, Basel } \\
\text { www.karger.com/cop }\end{array}$ \\
\hline
\end{tabular}

Tran et al.: An Unusual Case of Extranodal Rosai-Dorfman Disease Manifesting as an Epibulbar Mass
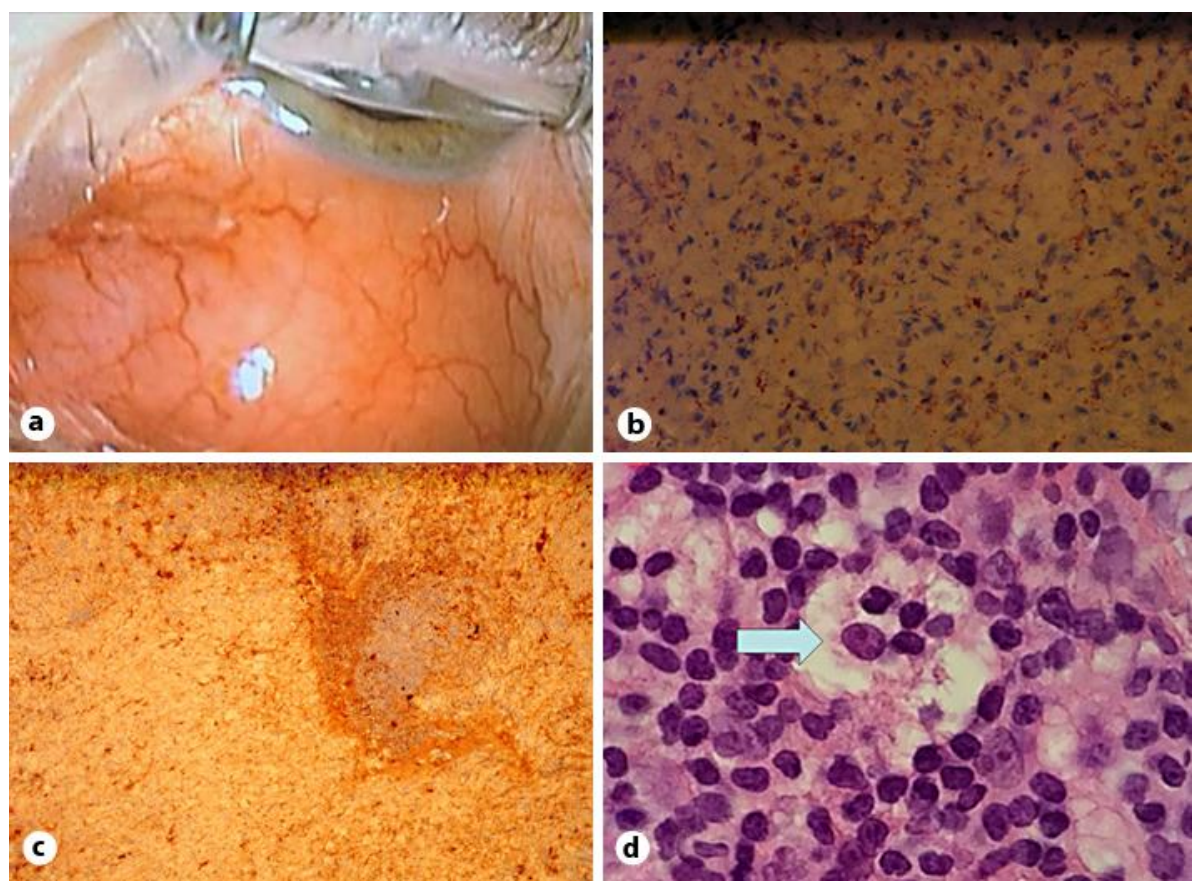

Fig. 1. Histopathology of the epibulbar mass. a Intraoperative photo of the salmon-colored conjunctival mass. b Moderate cytoplasmic staining with CD68. c Strong S100 stain uptake identifying histiocytes and the absence of lymphocyte staining in the center. $\mathbf{d}$ Arrow denotes emperipolesis with hematoxylin and eosin stain. 